\title{
Lumbàlgia crònica inespecífica. Tests físics per detectar-la. Prova pilot
}

\author{
Chronic Nonspecific Low Back Pain: Physical Tests to Detect It. \\ Pilot Study
}

JÚLIA JUBANY GÜELL

Escola universitària de Ciències de la Salut (UAB)

LISÍMACO VALLEJO CUÉLLAR JOAN RAMON BARBANY CAIRÓ

\author{
Autora per a la correspondència \\ Júlia Jubany Güell \\ jjubany@fub.edu
}

\section{Resum}

Objectiu: Valorar la capacitat de detecció de la lumbàlgia crònica inespecífica que tenen els indicadors: proporcionalitat dels temps de resistència entre extensors, flexors i musculatura lateral; temps de resistència dels extensors, Sorensen test i la fatigabilitat mitjançant electromiografia (EMG) per superfície. Igualment, valorar la influència sobre aquests indicadors que tenen les variables gènere, talla i pes. Disseny: Estudi pilot amb grup de control. Mesura mitjançant tres tests i EMG d'individus amb lumbàlgia crònica inespecífica i d'individus sense. La fiabilitat queda mesurada amb la repetició dels tests i de l'EMG en dies diferents. Participants: Tretze estudiants de fisioteràpia entre 18 i 32 anys. Set amb lumbàlgia crònica inespecífica i sis sense. Resultats: No s'han trobat diferències significatives entre la proporcionalitat dels temps de resistència entre extensors, flexors i musculatura lateral entre els individus que presentaven lumbàlgia i els que no en presentaven. Igualment, el Sorensen test i la potència mitjana obtinguda mitjançant EMG per superfície no han mostrat diferències entre els mateixos dos grups. El gènere ha resultat ser un element diferenciador respecte dels valors de les proporcionalitats dels temps de resistència de la musculatura extensora, flexora i musculatura lateral. El pes en el temps de resistència de la musculatura extensora ha resultat tenir una correlació de Pearson negativa $(r=-0,61)$. Conclusions: No s'han pogut diferenciar els individus amb lumbàlgia dels que no en presenten per a cap dels indicadors utilitzats. El pes i el gènere són variables que influeixen en aquests i que per tant s'han de tenir en compte durant l'avaluació dels individus mitjançant aquests tests.

Paraules clau: lumbàlgia crònica inespecífica, electromiografia, diagnòstic, proves físiques, Sorensen test

\begin{abstract}
Chronic Nonspecific Low Back Pain: Physical Tests to Detect It. Pilot Study

Objective: to assess the ability to detect chronic nonspecific low back pain with the following indicators: proportionality of endurance time for extensor, flexor and side muscles; Sorensen test of endurance time of extensor muscles; and fatigue using surface electromyography (EMG). Also to assess the influence of gender, height and weight variables on these indicators. Design: pilot study with a control group. Measured by three tests and EMG of individuals with nonspecific chronic low back pain and individuals without it. Reliability is measured by repetition of tests and EMG on different days. Participants: thirteen physiotherapy students aged between 18 and 32. Seven of them have chronic non-specific low back pain and six do not. Results: there were no significant differences between the proportionality of endurance time for extensor, flexor and side muscles between the individuals who have low back pain and those who do not. Likewise, the Sorensen test and average power obtained by surface EMG showed no differences between the two groups. Gender has proved to be a differentiating factor with respect to the proportionality values of endurance time for extensor, flexor and side muscles. Weight has had a negative Pearson correlation $(r=-0.61)$ in extensor muscle endurance time. Conclusions: it has not been possible to distinguish between those individuals who have low back pain and those who do not with any of the indicators used. Weight and gender are variables that affect these indicators and therefore should be taken into account when assessing individuals using these tests.
\end{abstract}

Keywords: chronic nonspecific low back pain, electromyography, diagnosis, physical tests, Sorensen test 


\section{Introducció}

El dolor lumbar és una síndrome d'etiologia multifactorial que afecta el $70-80 \%$ de la població com a mínim una vegada al llarg de la vida (Dieën, Selen, \& Cholewicki, 2003) (cfr. Candotti et al., 2008). Altrament anomenada lumbàlgia crònica inespecífica per la falta de coneixement de l'element causal del dolor, és una síndrome per a l'avaluació i/o control de la qual en moltes ocasions costa de trobar indicadors. Estudis actuals ja han demostrat la fatigabilitat de la musculatura extensora (detectada amb electromiografia per superfície) com a indicador d'aquesta lumbàlgia (Candotti et al., 2008; Farina, Gazzoni, \& Merletti, 2003; Kankaanpää, Taimela, Laaksonen, Hänninen, \& Airaksinen, 1998; Mannion, Connolly, Wood, \& Dolan, 1997; Peach \& McGill, 1995; Roy \& Oddsson, 1998). D'altres han buscat la relació d'aquesta lumbàlgia amb la pèrdua de resistència de la musculatura extensora (Sorensen test) (Hultman, Nordin, Saraste, \& Ohlsèn, 1993; Nicolaisen, 1985; Stephanopoulos, 1984) (cfr. Kankaanpää et al., 1998; Holmström, Moritz, \& Andersson, 1992). Finament, i amb un sol autor que hi dóna suport (McGill, 2007), un tercer indicador el trobem en l'alteració de les proporcionalitats que es deriven de la divisió dels temps obtinguts en quatre tests de resistència muscular: test de resistència de la musculatura extensora (TRME) o altrament conegut com a Sorensen test (figura 1); test de resistència de la musculatura flexora (TRMF) (figura 2); test de resistència de la musculatura lateral dreta (TRMLD) (figura 3), i test de resistència de la musculatura lateral esquerra (TRMLE). Les proporcionalitats descrites per McGill són les següents:

$$
\begin{array}{ll}
T R M F \div T R M E & \text { TRMFLD } \div \text { TRME } \\
T R M L F E \div T R M E & \text { TRMLD } \div \text { TRMLE }
\end{array}
$$

Per altra banda, existeix evidència clínica de la millora de la lumbàlgia crònica inespecífica després de la realització de diferents protocols d'exercici físic (Hurley et al., 2009; Marshall \& Murphy, 2006) que ens porta a pensar que les lumbàlgies cròniques poden estar associades a una pèrdua de condició física i/o muscular.

\section{Materials i mètodes Mostra}

Tretze estudiants de fisioteràpia d'entre 18 i 32 anys, 7 amb lumbàlgia crònica inespecífica i 6 sense. Del grup amb lumbàlgia, 3 són homes i 4 són dones; del grup sense lumbàlgia, 3 són homes i 3 són dones. La determinació de la mostra es va portar a terme segons casos típics a partir d'una enquesta passada prèviament a 286 persones (estudiants de fisioteràpia). Els subjectes candidats a participar a l'estudi eren aquells que mitjançant l'enquesta mostraven les característiques següents.

Grup amb lumbàlgia: presència de lumbàlgia crònica (dolor o desconfort localitzat per sota de la costella 12 i per sobre del plec gluti, amb dolor referit a la cama o sense, de dotze setmanes o més d'evolució) (Comunitat Europea, 2005) amb una freqüència alta d'aparició d'aquest dolor en l'últim any (només s'escollien les persones que havien respost els tres paràmetres de major freqüència de dolor lumbar entre els set proposats: "mai"; "una sola vegada"; "poques vegades i de forma molt esporàdica"; "força vegades"; "tot sovint"; "és una cosa molt habitual"; "sempre en tinc, convisc amb el dolor").

Grup sense lumbàlgia: totes aquelles persones que no havien sentit mai dolor lumbar.

Es van excloure totes aquelles persones que estiguessin en una fase aguda o reaguditzada de la seva lumbàlgia (rang de mobilitat de columna no funcional per a la vida diària a causa del dolor), totes aquelles persones que tinguessin una patologia de columna diagnosticada que expliqués el seu dolor, totes aquelles persones que havien estat sotmeses a intervencions quirúrgiques de columna o malucs i totes aquelles que tinguessin patologies que podien interferir en unes proves físiques (fibromiàlgia, miopatia...).

Dels subjectes candidats a participar en l'estudi, es van escollir de forma aleatòria els 13 que formen la mostra. Finalment, a tots els participants se'ls va demanar el consentiment informat per escrit i se'ls va assegurar la confidencialitat de les dades.

\section{Mètode per valorar els temps de resistència de la musculatura extensora, flexora i flexora lateral}

Els temps de resistència de les diferents musculatures es van valorar mitjançant els tests TRME, TRMF, TRMFLD i TRMFLE, que es van portar a terme durant una mateixa tarda a tots els individus de la mostra. Els subjectes van ser citats per parelles cada mitja hora i es va donar un temps de descans entre test i test d'aproximadament 15 minuts. Per valorar la fiabilitat dels tests, es va repetir el mateix protocol de la primera tarda en dues ocasions més (registres: dia $1=19 / 4$, dia $2=21 / 4$, i dia $3=23 / 4)$. A continuació es detallen els quatre tests: 


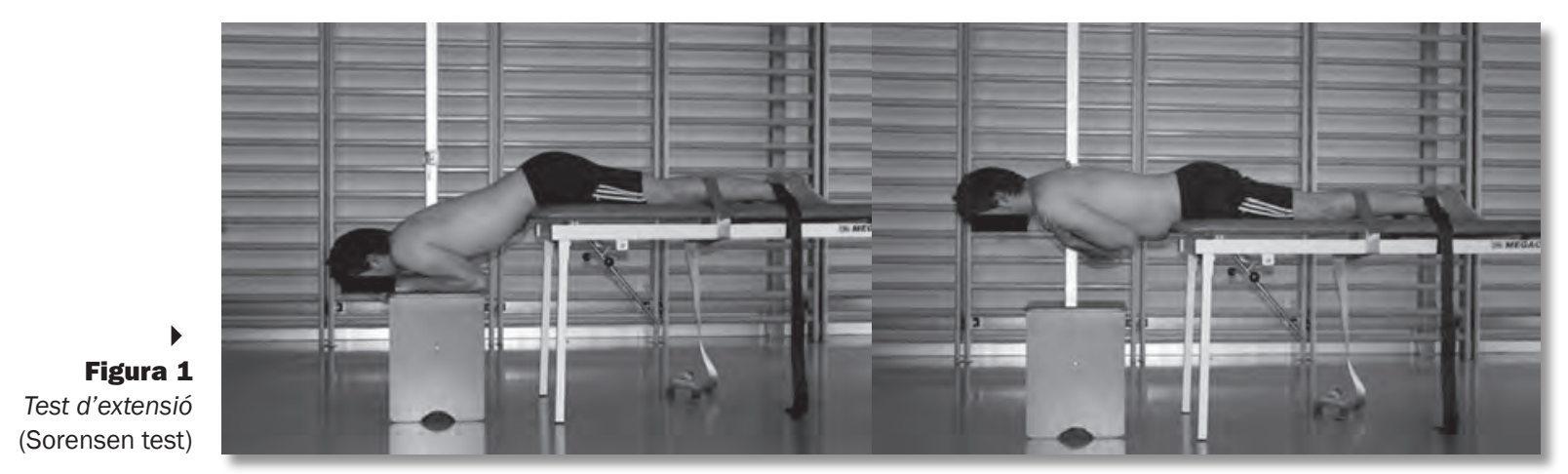

- Test de resistència de la musculatura extensora "Sorensen test") (fig. 1): Individu sobre la llitera en decúbit pron amb el tronc fora d'aquesta i els braços recolzats sobre un tamboret que li permet sustentar-se sense activar la musculatura extensora. Les crestes ilíaques es troben a sobre $\mathrm{i}$ alineades amb el marge de la llitera. La part restant del cos es troba fixada a la llitera mitjançant unes corretges localitzades al nivell de la part més prominent del bessó i el turmell. Es demana a l'individu que abandoni el recolzament dels braços encreuant-los al pit i que elevi el tronc fins a la seva alineació amb les extremitats inferiors. Es demana a l'individu que mantingui aquesta posició fins que no pugui controlar-la més o que aquesta esdevingui intolerant. Per estandarditzar el nivell d'elevació del tronc durant la realització del test, s'avalua prèviament en cada individu l'alçada en què es troba la part més prominent (dorsal) de tòrax (escàpules o columna) quan l'individu està totalment recolzat en la mateixa llitera en decúbit pron. Aquesta, doncs, és l'alçada que es demana en el test. L'indicador d'alçada que s'utilitza és una barra horitzontal subjectada a una estructura vertical que permet l'ascens i descens sobre aquesta $i$, a la vegada, ser fixada en el punt que convingui segons cada individu. Es demana al subjecte que durant tota la durada del test no perdi el con- tacte amb aquesta estructura. El test mesura els segons en què l'individu és capaç d'aguantar la posició horitzontal al terra amb les mans encreuades al pit. El cronòmetre es para en el moment que l'individu perd l'horitzontalitat.

- Test de resistència de la musculatura flexora (fig. 2): Subjecte en sedestació sobre la llitera, amb la part superior del cos recolzada sobre un suport a $60^{\circ}$ de l'horitzontal i els genolls i malucs amb una flexió de $90^{\circ}$. Les extremitats inferiors es troben recolzades a la llitera només a nivell dels peus i aquests, a la vegada, es troben fixats amb una corretja a la mateixa llitera. Amb els braços encreuats al pit i la barra horitzontal (ja utilitzada en el test anterior) com a punt de referència per al pacient a nivell de la segona-tercera costella, es demana al subjecte que mantingui la posició del cos i el contacte amb la barra horitzontal mentre se li retira el suport de l'esquena. Es comptabilitza el temps que aquest és capaç de mantenir la postura i el contacte amb la barra horitzontal. El test s'acaba quan això ja no li sigui possible (McGill, Childs, \& Liebenson, 1999).

- Test de resistència de la musculatura flexora lateral (side bridge test) dreta (fig. 3): Subjecte en decúbit lateral sobre la llitera amb les cames estirades (el peu de la cama de sobre recolzat just

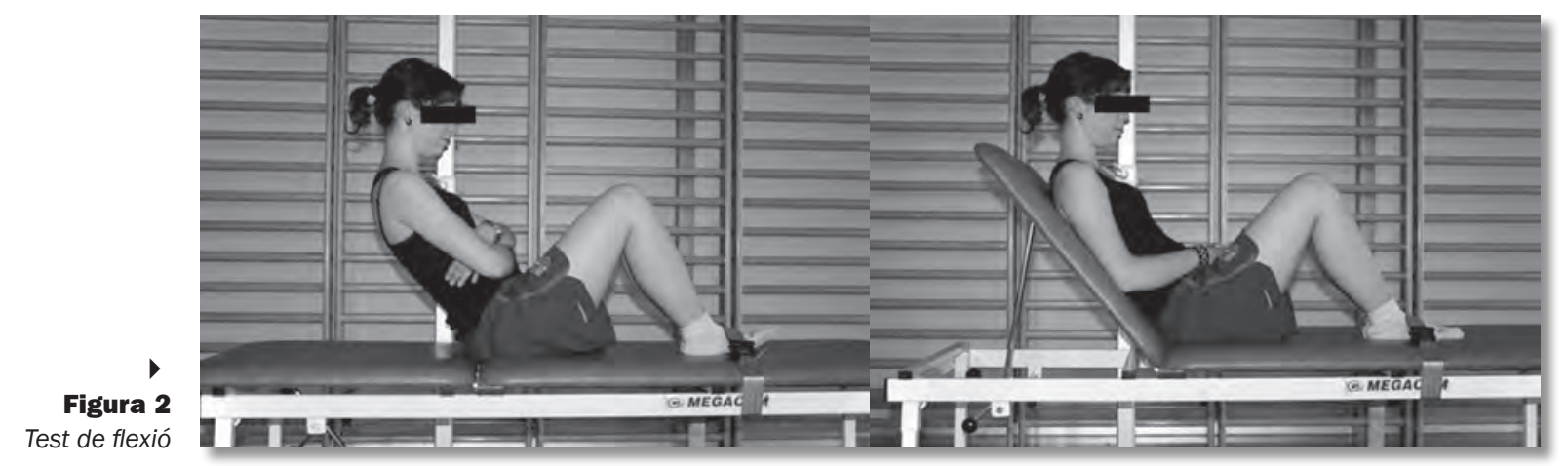




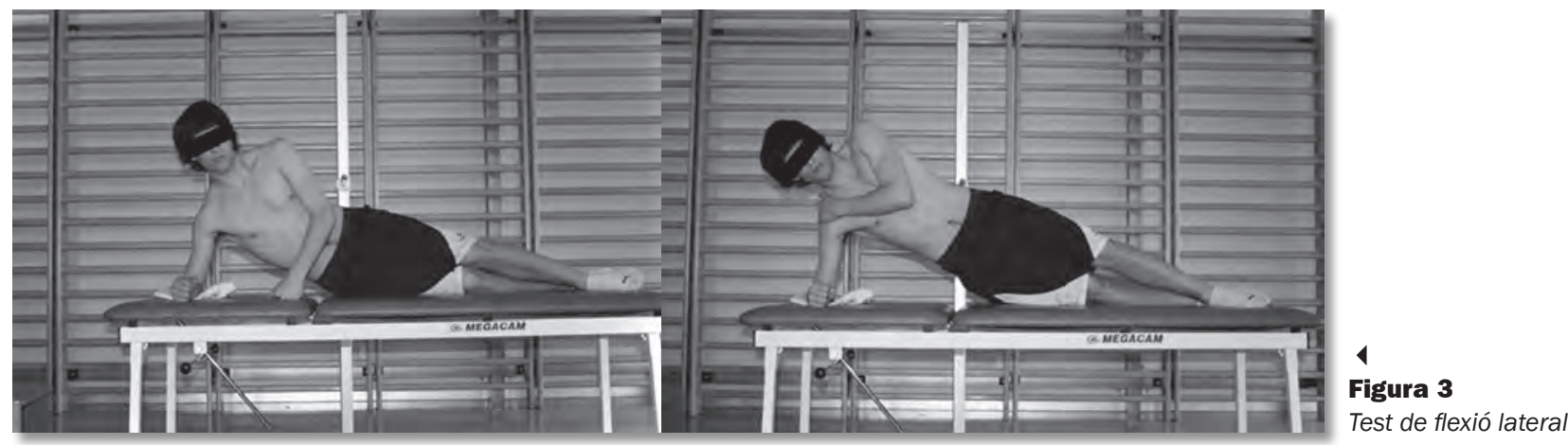

per davant del de sota) i l'avantbraç recolzat amb el colze just a la vertical de l'espatlla. Es demana al subjecte que, tot perdent el contacte del maluc amb la llitera, adopti una postura d'alineació del tronc i les extremitats inferiors i únicament recolzi els peus i el colze homolateral. El braç no involucrat en el recolzament es col-loca encreuat al pit, amb la mà sobre l'espatlla contrària. El test s'acaba quan es perd la postura descrita $\mathrm{i}$ es torna al recolzament inicial (McGill et al., 1999). Per controlar més la posició, aquí també es col·loca la barra horitzontal. En aquesta ocasió es col-loca a nivell del maluc, just a l'alçada on la col-locació de l'individu és una alineació dels seus segments corporals (cap, tronc i extremitats inferiors). Es demana, com en els casos anteriors, que l'individu no perdi el contacte amb aquesta.

- Test de resistència de la musculatura flexora lateral (side bridge test) esquerra: Mateix test que en l'apartat anterior, però el subjecte es troba recolzat en el costat contrari.

Els indicadors utilitzats per a la detecció de la lumbàlgia crònica van ser les diferents proporcionalitats que ens resulten de la divisió dels temps obtinguts en cada test entre el temps obtingut en el Sorensen test per si sol, tal com s'indica a continuació:

- Indicador 1: TRMF $\div$ TRME (segons)

- Indicador 2: TRMFLD $\div$ TRME (segons)

- Indicador 3: TRMLFE $\div$ TRME (segons)

- Indicador 4: TRMLD $\div$ TRMLE (segons)

- Indicador 5: Sorensen test per si sol (segons)

\section{Mètode per l'EMG: fatigabilitat de la musculatura extensora}

Per enregistrar el senyal electromiogràfic de la musculatura extensora, es va utilitzar el sistema electromiogràfic ME6000 (Mega Electronics, Kuo- pio, Finlàndia), amb elèctrodes adhesius de superfície $(\mathrm{Ag} / \mathrm{AgCl})$ d'1 $\mathrm{cm}^{2}$, a continuació de l'aplicació d'un gel conductor (Ambu Blue Sensor, M-00-S, Dinamarca). Els elèctrodes van ser col-locats de forma paral·lela a les fibres musculars i amb una distància entre elèctrodes de $2,5 \mathrm{~cm}$. Abans de la col·locació d'aquests es va preparar la zona de treball tot rasurant el pèl i netejant la pell amb alcohol (Coorevits, Danneels, Cambier, Ramon, \& Vanderstraeten, 2008). Es van col-locar els elèctrodes a la següent musculatura i de forma bilateral: musculatura erectora espinal, part toràcica $(2 \mathrm{~cm}$ lateral, a T-12, amb els elèctrodes col·locats de forma paral·lela a les fibres del múscul) (Cram \& Kasman, 1998); musculatura erectora espinal, part lumbar $(2 \mathrm{~cm}$ lateral, a $\mathrm{L} 3$, amb els elèctrodes col-locats de forma paral-lela a les fibres del múscul) (Cram \& Kasman, 1998); gluti major (punt mig entre el trocànter major i les vèrtebres sacres, amb una direcció obliqua caudal i lateral lleugerament més superior que el trocànter) (Cram \& Kasman, 1998); i bíceps femoral (punt mig de la línia que uneix la tuberositat isquiàtica amb l'epicòndil lateral de la tíbia) (Freriks \& Hermens, 2000).

El senyal en cru va ser enregistrat com a freqüència de mostreig de $1.000 \mathrm{~Hz}$, i es va utilitzar la mitjana quadràtica (root mean square-RMS) en intervals de $0,05 \mathrm{~s}$. El preamplificador es va localitzar a $6 \mathrm{~cm}$ dels elèctrodes (sensibilitat d' $1 \mathrm{mV}$, augment de 305 , rang de freqüències 8-500 Hz). El paràmetre d'EMG usat va ser la potència mitjana $(\mathrm{PM}: \mu \mathrm{V})$.

Es va prendre, en un primer moment, un registre de 10 segons de la musculatura en repòs. La posició de repòs es descriu com a decúbit pron amb els braços recolzats sobre la llitera per damunt del cap i amb el cap recolzat de costat mirant cap a la dreta. Posteriorment es va demanar a l'individu la realització del Sorensen test, on es va prendre un altre registre electromiogràfic de tota la durada d'aquest 


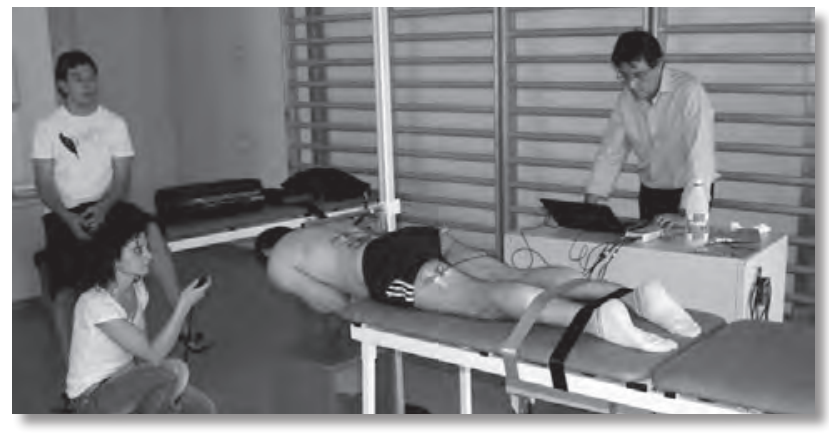

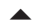

\section{Figura 4}

Electromiografia per superfície

(fig. 4). Un cop finalitzat el test, es va demanar al subjecte que es tornés a posar en la mateixa posició de repòs per tornar a prendre un últim registre: repòs postesforç. Tal com es pot veure en l'apartat de resultats, no es va poder enregistrar el gluti esquerre per problemes tècnics amb l'equip.

Per valorar la fiabilitat del procediment de l'enregistrament electromiogràfic en el test d'extensió Sorensen test, es van fer enregistraments en dos individus (dues noies de 23 i 27 anys) en dos dies no consecutius. Es van enregistrar els següents paràmetres per valorar si en dies diferents s'obtenien resultats equivalents: mitjà, mínim, màxim, àrea i mediana del pic de potència.

Durant la realització de tots els tests es va animar el subjecte a mantenir la posició sense una excessiva eufòria i intentant sempre fer-ho amb la mateixa insistència i només sota el guiatge de l'investigador. Als subjectes no se'ls va permetre saber els seus resultats per no condicionar-los. Igualment no se'ls va explicar l'objectiu de l'estudi. El dia de la realització dels tests es van mesurar i pesar tots els individus.

\section{Anàlisi estadística}

Es va fer una anàlisi estadística de les dades a partir del càlcul de les mitjanes i desviacions estàndards de totes les variables. La normalitat de la distribució de les variables va ser analitzada mitjançant el test de ShapiroWilk $(n<50)$. El coeficient de correlació de Pearson va ser utilitzat per determinar les relacions entre els paràmetres seleccionats durant els tests. Quan va ser necessari es va fer una anàlisi de regressió lineal i es va calcular l'error estàndard de l'estimació (SEE). Per determinar el grau d'estabilitat (concordança) dels valors durant els tests (t1 vs. t2 vs. t3), es va utilitzar el coeficient de correlació de Pearson (r), el coeficient de correlació intraclasse (CCI) i una ANOVA de mesures repetides (model lineal generalitzat). Tota l'anàlisi estadística va ser calculada mitjançant el programa estadístic SPSS per a Windows versió 15.0 (SPSS Inc., USA).

\section{Resultats}

\section{Enquestes}

El nombre total d'enquestes contestades és de 286 , de les quals 122 són d'homes i 164 són de dones. Segons la freqüència de dolor lumbar que patien els entrevistats, trobem: "mai": 35; "una sola vegada": 10; "poques vegades i de forma molt esporàdica": 134; "força vegades": 62; "tot sovint": 24; "és una cosa molt habitual": $14 \mathrm{i}$ "sempre en tinc, convisc amb el dolor": 5 (fig. 5).

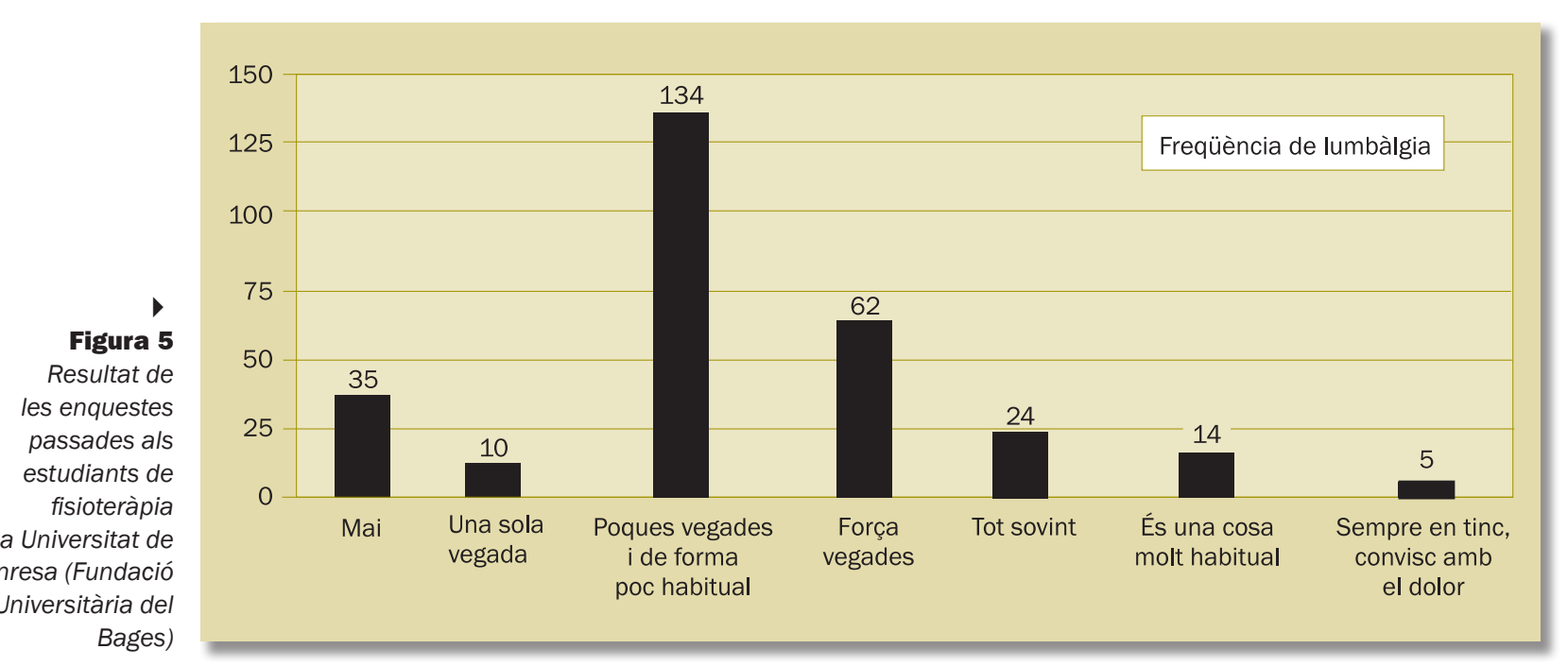




\section{Fatigabilitat de la musculatura extensora}

En l'anàlisi de variància ANOVA de mesures repetides entre el grup amb presència de lumbàlgia i el que no en presenta, no s'observa cap grau de significança per sota del 0,05 .

En cap dels músculs la PM dels períodes de repòs pretest i posttest no és significativament diferent, i per això es pot concloure que l'activació muscular del període de repòs previ al test és similar a l'activació del període de repòs posterior al test (o temps de recuperació muscular).

Finalment, en la PM s'han trobat diferències significatives entre la musculatura erectora espinal de nivell L3 dreta i l'esquerra en un valor $p=0,02$, en tota la mostra, $n=5$.

\section{Discussió}

En aquest estudi pilot no s'han pogut diferenciar les persones amb lumbàlgia de les que no en presenten, amb cap dels tres mètodes -proporcionalitat dels temps de resistència entre extensors, flexors i musculatura lateral; temps de resistència de la musculatura extensora, i fatigabilitat mitjançant EMG-, tal com s'havia plantejat en un inici i com deia la literatura (Candotti et al., 2008; Farina et al., 2003; Holmström et al., 1992; Kankaanpää et al., 1998; Mannion et al., 1997; McGill, 2007; Peach \& McGill, 1995; Roy \& Oddsson, 1998). Sí que s'han trobat relacions entre les variables gènere, pes i talla en alguns dels tres anteriors mètodes, tal com es mostra a continuació.

Pel que fa al quocient entre qualsevol test i el Sorensen test, els homes obtenen un resultat significativament més alt que les dones. Dit d'una altra manera, els homes tenen les musculatures tant flexora com flexora lateral més resistents que les dones (comparativament amb la seva musculatura extensora). Es conclou, doncs, que s'haurien de presentar models diferents per als homes i per a les dones en la detecció de lumbàlgia crònica inespecífica mitjançant el mètode de les proporcions.

La variable del pes ha resultat ser influent només sobre un dels tests. Ha mostrat una correlació negativa $(r=-0,61)$ amb el test de resistència de la musculatura extensora (Sorensen test). Com més pesa l'individu menys temps de resistència aconsegueix en el test (fig. 6). Si es volgués extreure una relació entre el Sorensen test $\mathrm{i}$ la presència de lumbàlgia $\mathrm{o}$ no, caldria tenir en compte aquesta variable. Pel que fa a la resta de tests i les proporcions, la variable pes no ha mostrat tenir correlació, igualment que la variable talla.

Segons aquest estudi, no hi ha diferències significatives entre l'activitat del múscul en repòs previ al test i l'acti- vitat en repòs posterior a la realització del test. Els petits canvis existents es relacionen més amb els petits canvis en la postura (per més que aquesta ja estigui preestablerta $\mathrm{i}$ controlada) que no amb si el múscul ha fet o no l'esforç.

Pel que fa als resultats comparatius entre la musculatura dreta i l'esquerra en la població analitzada, existeixen diferències significatives en les PM entre la musculatura erectora espinal del nivell L3 dreta i l'esquerra, i no en les altres musculatures enregistrades per a la mostra total. Es podria pensar que una asimetria molt accentuada de la musculatura podria portar a un desequilibri i ser causa de lumbàlgia. Caldria, però, més recerca en aquesta línia.

Respecte a la valoració de la fiabilitat dels tests utilitzats en la valoració de les proporcionalitats i el procés d'enregistrament de l'EMG per superfície, es pot concloure que aquests han demostrat tenir un alt grau de fiabilitat a partir de l'anàlisi mitjançant una ANOVA de mesures repetides i el CCI.

Mitjançant les enquestes, s'ha evidenciat l'alta presència de lumbàlgia en la població jove, tot i que en un primer instant hom pensaria que aquesta població hauria de ser sana. Són dades que no s'allunyen de la realitat ja analitzada en la literatura (Dieën et al., 2003) (cfr. Candotti et al., 2008). La lumbàlgia és una disfunció molt habitual en la població en general, però tal com podem observar en les dades d'aquest estudi, també en la població jove.

Un biaix que pot haver condicionat aquest estudi és el fet de tractar amb la variable "presència de lumbàlgia crònica inespecífica". La lumbàlgia crònica es una síndrome multifactorial, amb un alt component de subjectivitat en la seva vivència i una alta vinculació a la somatització. Es tracta d'una variable en la qual múltiples factors de difícil control poden intervenir-hi (estat emocional, vivències prèvies de la persona, creences, cultura...). Així, doncs, no és fàcil tenir una mostra homogènia de persones amb lumbàlgia, ja que aquesta pot ser de característiques o orígens molt variables, i això fa que les lumbàlgies potser no siguin comparables.

Cal ser conscients que el fet de ser una prova pilot, i per tant utilitzar una mostra relativament baixa $(n=13$ per als test de resistència i $n=5$ per al registre electromiogràfic), ha permès que amb la seva realització s'hagi pogut comprovar la viabilitat de l'estudi tot i que els resultats hagin estat d'una validesa externa molt baixa. Es considera que aquest estudi pilot és un pas previ a la realització d'un estudi amb les mateixes característiques però amb una mostra més gran (60-80 subjectes), i es creu que els resultats exposats anteriorment podrien variar molt en la realització d'aquest altre estudi. 
Es creu també que en un futur estudi amb una mostra major caldria una anàlisi estadística més completa, que utilitzi com a indicador de fatigabilitat no només la PM sinó la freqüència mediana, la freqüència mitjana $i$ l'amplitud de l'espectre en la meitat de l'amplitud del pic (half-width), paràmetres als quals alguns autors donen suport per a la detecció de lumbàlgia crònica inespecífica tot i que amb resultats contradictoris (De Luca, 2006; Farina et al., 2003; Humphrey, Nargol, Jones, Ratcliffe, \& Greenough, 2005). Per altra banda, amb una mostra major també es podria avaluar si les persones amb lumbàlgia tenen registres electromiogràfics diferents en un mateix múscul però en costats diferents del cos, o avaluar si el pes, la talla i el gènere influeixen en el test d'EMG, ja que són aspectes que no s'han tractat en aquest estudi per la baixa mostra i pel fet de ser un estudi pilot.

\section{Conclusions}

Les conclusions que podem extreure d'aquest estudi pilot són:

- Amb la mostra utilitzada no s'han pogut diferenciar les persones amb lumbàlgia de les que no en presenten en cap dels mètodes utilitzats: proporcionalitat dels temps de resistència entre extensors, flexors $\mathrm{i}$ musculatura lateral, temps de resistència de la musculatura extensora (Sorensen test), i la fatigabilitat mitjançant EMG, tal com s'havia plantejat a l'inici.

- El pes i el gènere són variables que influeixen en els tests i que per tant s'han de tenir en compte. El pes influeix sobre el Sorensen test i la variable gènere, sobre les proporcionalitats entre els tests.

- La talla no ha mostrat influència en els tests pel que fa a la mostra analitzada en aquest estudi.

- Cal una mostra major per poder extrapolar els resultats a la població en general.

\section{Referències}

Candotti, C. T., Loss, J. F., Pressi, A. M. S., De Souza, F. A., La Torre, M.; De Oliveira, M., ... \& Pasini, M. (2008). Electromyography for Assessment of Pain in Low Back Muscles. Physical Therapy, 88(9), 1061-1067. doi:10.2522/ptj.20070146

Comunitat Europea (2005). Versió espanyola de la Guía de Práctica Clínica del Programa Europeo COST B13 "Low back pain: Guidelines for its manageement". European Commission Directorate General Research.

Coorevits, P., Danneels, L., Cambier, D., Ramon, H., \& Vanderstraeten, G. (2008). Assessment of the validity of the Biering-Sørensen test for measuring back muscle fatigue based on EMG median frequency characteristics of back and hip muscles. Journal of Electromyography and Kinesiology, 18(6), 997-1005. doi:10.1016/j.jelekin.2007.10.012

Cram, J. R., \& Kasman, G. S. (1998). Introduction to surface electromiography. USA: Aspen publishers.

De Luca, C. J. (2006). Electromyography. A John G. Webster (Ed.),
Encyclopedia of Medical Devices and Instrumentation. John Wiley.

Dieën, J. H. van, Selen, L. P., \& Cholewicki, J. (2003). Trunk muscle activation in low-back pain patients: an analysis of the literature. Journal of Electromyography and Kinesiology, 13(4), 333-251. doi:10.1016/S1050-6411(03)00041-5

Farina, D., Gazzoni, M., \& Merletti, R. (2003). Assessment of low back muscle fatigue by surface EMG signal analysis: methodological aspects. Journal of Electromyography and Kinesiology, 13(4), 319-332. doi:10.1016/S1050-6411(03)00040-3

Freriks, B., \& Hermens, H. J. (2000). SENIAM projecte: European Recommendations for Surface electroMyoGraphy: Roessingh Research and Development.

Holmström, E., Moritz, U., \& Andersson, M. (1992). Trunk muscle strength and back muscle endurance in construction workers with and without low back disorders. Scandinavian Journal of Rehabilitation Medicine, 24(1), 3-10.

Hultman, G., Nordin, M., Saraste, H., \& Ohlsèn, H. (1993). Body composition, endurance, strength, cross-sectional area, and density of $\mathrm{mm}$ erector spinae in men with and without low back pain. Journal of Spinal Disorders, 6(2), 114-123. doi:10.1097/00002517-199304000-00004

Humphrey, A. R., Nargol, A. V. F., Jones, A. P. C., Ratcliffe, A. A., \& Greenough, C. G. (2005). The value of electromyography of the lumbar paraspinal muscles in discriminating between chronic-lowback-pain sufferers and normal subjects. European Spine Journal, 14(2), 175-184. doi:10.1007/s00586-004-0792-3

Hurley, D. A., O'Donoghue, G., Tully, M.A., Moffett, J. K., Van Mechelen, W., Daly, L., ... McDonough, S. M. (2009). A walking programme and a supervised exercise class versus usual physiotherapy for chronic low back pain: a single-blinded randomised controlled trial. (The Supervised Walking in comparison to Fitness Training for Back Pain -SWIFT_- Trial). BMC Musculoskelet Disord, 10: 79. doi:10.1186/1471-2474-10-79

Kankaanpää, M., Taimela, S., Laaksonen, D., Hänninen, O., \& Airaksinen, O. (1998). Back and hip extensor fatigability in chronic low back pain patients and controls. Archives of Physical Medicine and Rehabilitation, 79(4), 412-417. doi:10.1016/S0003-9993(98)90142-3

Mannion, A., Connolly, B., Wood, K., \& Dolan, P. (1997). The use of surface EMG power spectral analysis in the evaluation of back muscles function. Journal of Rehabilitation Research and Development, 34(4), 427-439.

Marshall, P. W. M., \& Murphy, B. A. (2006). Evaluation of Functional and Neuromuscular Changes after Exercise Rehabilitation for Low Back Pain Using a Swiss Ball: A Pilot Study. Journal of Manipulative and Physiological Therapeutics, 29(7), 550-560. doi:10.1016/j. jmpt.2006.06.025

Martín, R., Fernández, M., Veiga, J. V., Otero, J., \& Rodríguez, F. A. (2001). Fiabilidad de las pruebas de fuerza en salto vertical y velocidad de carrera en escolares de 6 a 8 años. Apunts. Educación Física y Deportes (63), 40-45.

Martínez, M. A., Sánchez, A., \& Faulin, J. (2008). Bioestadística amigable. Ediciones Díaz de Santos.

McGill, S. M. (2007). Low Back Disorders (2. ${ }^{a}$ ed.). Canadá: Human Kinetics.

McGill, S. M., Childs, A., \& Liebenson, C. (1999). Endurance times for low back stabilization exercises: Clinical targets for testing and training from a normal database. Archives of Physical Medicine and Rehabilitation, 80(8), 941-944. doi:10.1016/S0003-9993(99)90087-4

Nicolaisen T.; J. K. (1985). Trunk strength, back muscle endurance and low-back trouble. Scandinavian Journal of Rehabilitation Medicine, 17(3), 121-127.

Peach, J. P., \& McGill, S. M. (1995). Classification of Low Back Pain with the Use of Spectral Electromyogram Parametres. Spine, 23(10), 1117-1123. doi:10.1097/00007632-199805150-00009

Roy, S. H., \& Oddsson, L. I. E. (1998). Classification of Paraspinal Muscle Impairements by Surface Electromyography. Physical Therapy, $78(8), 838-851$.

Stephanopoulos, G. (1984). Chemical Process Control. An introduction to theory and practice: New Jersey: Prentice/Hall International, Inc. 